\title{
Validation of high-energy nuclear models: State-of-the-art and perspectives
}

\author{
Sylvie Leray ${ }^{\mathrm{a}, *}$, Alain Boudard ${ }^{\mathrm{a}}$, Joseph Cugnon ${ }^{\mathrm{b}}$, Jean-Christophe David ${ }^{\mathrm{a}}$, \\ Jean-Eric Ducret ${ }^{\mathrm{a}}$, Eric Le Gentil ${ }^{\mathrm{a}}$, Stéphane Pietri ${ }^{\mathrm{a}}$, Claude Volant ${ }^{\mathrm{a}}$ \\ ${ }^{a} C E A / S a c l a y, D A P N I A / S P h N, 91191$ Gif-sur-Yvette, Cedex, France \\ ${ }^{\mathrm{b}}$ Université de Liège,allée du 6 août 17, Bât.B5, B-4000 Liège 1, Belgique
}

Available online 2 March 2006

\begin{abstract}
The state-of-the-art regarding the predicting capabilities of some of the physics models available in MCNPX for Accelerator-Driven System design is discussed. The identified remaining deficiencies, which originate either from a lack of experimental data or from not well understood physics mechanisms, are stressed. To solve these problems, more constraining experiments are now needed to allow a deeper understanding of the reaction mechanisms.
\end{abstract}

(C) 2006 Elsevier B.V. All rights reserved.

PACS: 25.40.-h; 25.40.Sc; 28.50.Qd

Keywords: Accelerator-driven systems; Proton-induced reactions; Spallation reactions; Nuclear models

\section{Introduction}

In the high-energy transport codes that are used to design Accelerator-Driven Systems (ADS) for nuclear waste transmutation or spallation neutron sources, elementary cross-sections and characteristics of all the reaction products are calculated by nuclear models above $200 \mathrm{MeV}$. During the last years, new high-quality experimental data have been collected, leading to a better understanding of the spallation reaction mechanism and allowing the testing of the currently used high-energy models. A large part of this work has been done in the framework of the HINDAS European FP5 program [1]. Also an important effort has been devoted to the development of more reliable spallation models, as the INCL4 [2] - ABLA [3] combination of intranuclear cascade and de-excitation models in Europe or CEM2k in USA [4], both now available in MCNPX [5].

As a result of this work, it is now possible to draw some conclusions on the degree of reliability of the predictions of

\footnotetext{
*Corresponding author. Tel.: + 33169088361 ; fax: + 33169087584 .

E-mail address: sleray@cea.fr (S. Leray).
}

quantities relevant for ADS. Some persisting discrepancies in the comparison between models and data have also been identified. However, it is not always easy to determine whether they originate from a lack of reliability of the experimental data or from deficiencies in the models. In the latter case, it is also often difficult to know which part of the model (intra-nuclear cascade or de-excitation stage) is responsible for the deficiency. To solve these problems, more constraining experiments are now being carried out, as NESSI [6] or SPALADIN experiments [7], that should allow a deeper understanding of the reaction mechanisms.

\section{State-of-the-art}

In [1], a comprehensive comparison between the predictions of mainly INCL4-ABLA and Bertini-Dresner [8,9] (default option of MCNPX) and the whole set of available experimental data has been carried out. It must be stressed that this was done with always the same set of (default) parameters in the models, whatever the system studied or the observable compared. Other comparisons [10] have been performed with other intranuclear cascade or deexcitation models. Although some important differences 
between the predictions of the models are found, some general trends can be observed.

\subsection{Neutron production}

As regards neutron production, which is of primary importance for neutron source design, the situation is rather clear. Thanks to the existing complete and coherent set of experimental data on double-differential crosssections and multiplicities on both thin and thick targets, it can now be stated that total neutron production in an individual spallation reaction as well as in a realistic ADS target can be predicted with a precision of $10-15 \%$, whatever the combination of intranuclear cascade and de-excitation models used in MCNPX. This may be due to the fact that, neutron production being of major importance for applications and the amount of data being rather large, most models have been first adjusted to reproduce this quantity. The prediction precision of the models corresponds also roughly to the uncertainties of the experimental data, meaning that it cannot be much improved. General trends of energy, angular or geometry (for thick targets) dependence are also well understood, although, locally, discrepancies could reach a few tens of percent.

\subsection{Light-charged particles}

A correct prediction of the production yields of hydrogen and helium isotopes is important for damage assessment in solid spallation targets, structure materials and window separating the accelerator from the target. While data on helium production obtained with different measurement techniques [11,12] agree for lead targets, not understood discrepancies remain for iron, as can be seen in Fig. 1. Comparisons with codes have revealed severe deficiencies in most of the currently used models. This is also shown in Fig. 1 in which it can be observed that both Bertini-Dresner and INCL4-ABLA systematically underpredict the experimental data. On the contrary, the replacing of ABLA by another de-excitation model, GEMINI [13], improves largely the situation. It should be added that GEMINI also generally reproduces the shape of the evaporation part of the particle spectra [11]. A further improvement is obtained with the new version of INCL4 [14] that allows for the formation of helium clusters by a surface coalescence mechanism during the cascade stage. This mechanism is necessary to explain the highenergy tail observed in the light-charged particle energy spectra. The resulting yield has to be added to the one from evaporation. It leads to a non-negligible enhancement of the total helium production which varies from $15 \%$ at the highest energy to $30 \%$ at $300 \mathrm{MeV}$. The decrease of the effect with increasing energy is due to the fact that helium emission during the cascade stage is not very dependent on energy while evaporation of helium strongly depends on excitation energy and thus on incident energy.

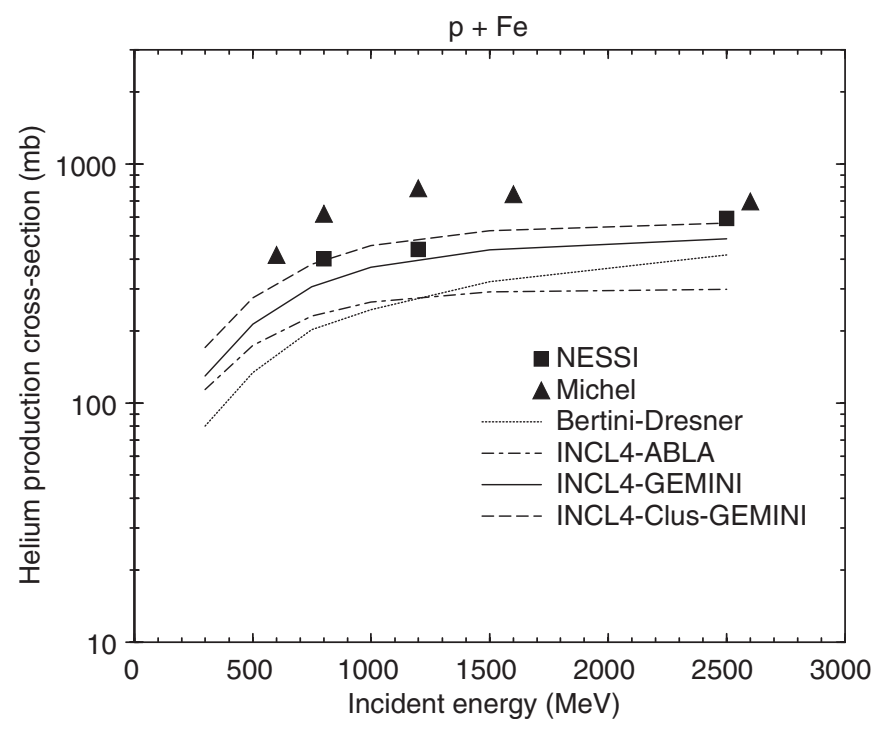

Fig. 1. Helium production measured by the NESSI group [11] and Michel et al. [12] compared to the predictions of Bertini-Dresner (dotted line) and INCL4 coupled either to ABLA (dashed-dotted line) or GEMINI (full line). The dashed curve is obtained with the version of INCL4 allowing helium cluster formation.

\subsection{Heavy residues}

The precise knowledge of spallation residues is important for assessment of radioactivity and damage. HINDAS has brought an extensive set of high-quality data thanks both to the reverse-kinematics technique, which has lead to the measurements of thousands of identified isotopes and to direct-kinematics experiments providing excitation functions [1]. These data showed that the Bertini-Dresner combination of models generally does not predict correctly the isotopic distributions (except for isotopes very close to the target nucleus) and, in the case of heavy systems, the fission fragment production. On the contrary INCL4-ABLA has been shown to give a satisfying agreement with isotopic production cross-sections of evaporation residues not too far from the target nucleus and in the region of fission. This is particularly important for the prediction of volatile element production, which is mostly due to fission in a liquid metal target. In fact, a recent experiment performed at ISOLDE [15] on a thick $\mathrm{Pb}-\mathrm{Bi}$ target has confirmed that $\mathrm{Kr}$ and $\mathrm{Hg}$ production is much better predicted with INCL4ABLA than Bertini-Dresner. On the other hand, the total target activity, which is mainly due to isotopes very close to the target, is rather well predicted by all codes [16].

However, a systematic misprediction of light evaporation residues has been observed with INCL4-ABLA and other standard models [2,4]. This is illustrated in Fig. 2 (red curve), which shows the charge distribution for iron measured by [17] at GSI at different energies. The same behaviour, although beginning for charges much farther from the target nucleus, was also noticed for heavy systems [2]. This under-prediction is not really understood since it could be due either to too low excitation energy at the end 


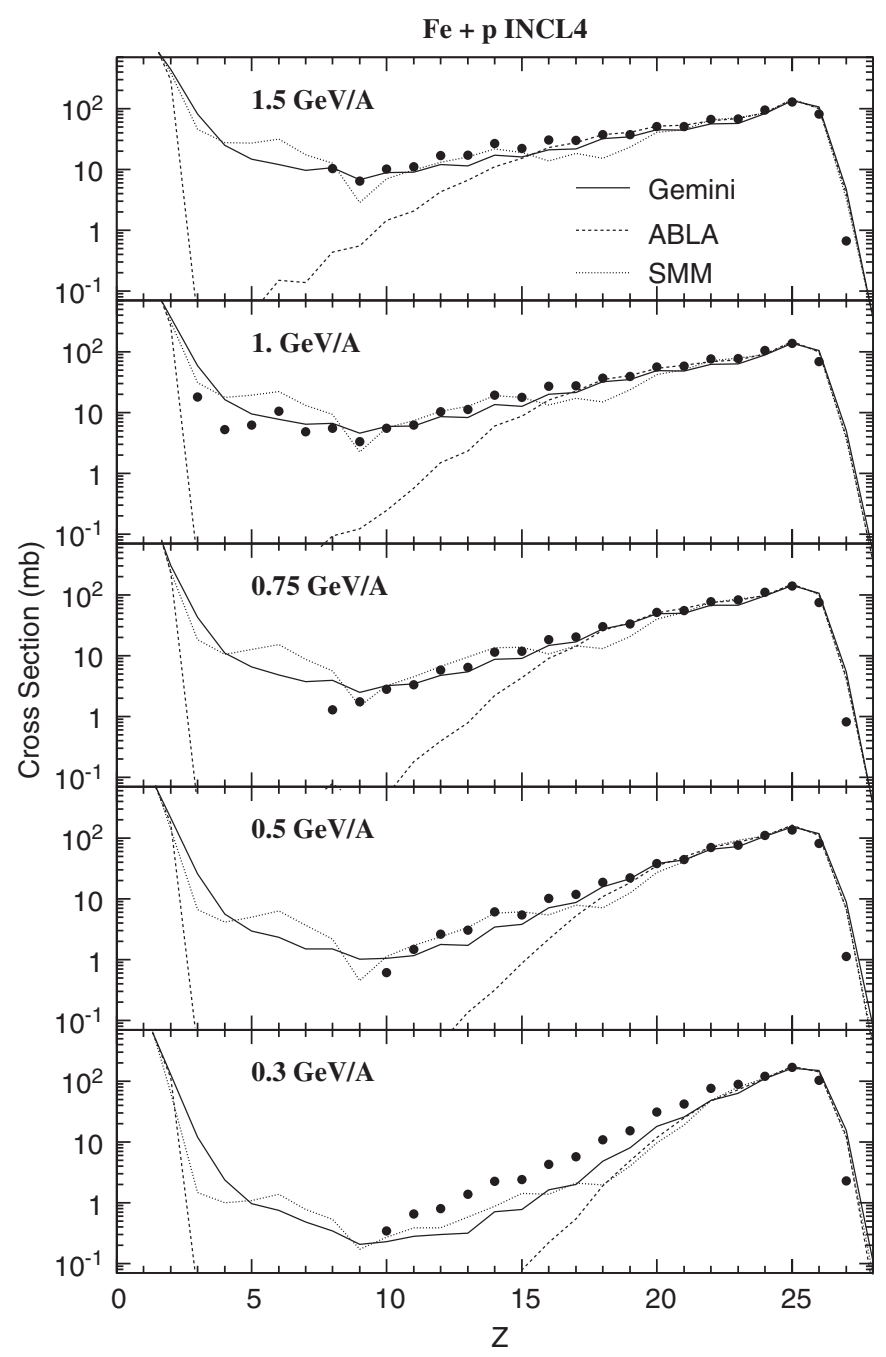

Fig. 2. Charge distributions at 5 energies [17], compared with the predictions of the INCL4 model coupled with the de-excitation codes ABLA [3], GEMINI [13] or SMM [18].

of the cascade stage or to deficiencies of the de-excitation model. In Fig. 2 we also show a comparison of the experimental data with the same INCL4 intranuclear cascade model but followed by the GEMINI (blue) and SMM (green) de-excitation codes. In the first model, intermediate mass fragments (IMF) can be produced through an asymmetrical fission mode competing with classical evaporation, while in SMM [18] it is due to multifragmentation. It can be seen that both calculations agree quite well with the whole charge distributions. GEMINI even reproduces the small even-odd effects observed in the data and the IMF production measured at $1 \mathrm{GeV}$. Also the isotopic distributions, not shown, are rather well predicted. A similar agreement of GEMINI following another INC model has been recently reported by Mashnik [4]. It has to be noticed however, that the same GEMINI model is not able to reproduce data on heavy nuclei. The need for an additional mechanism producing IMFs in the de-excitation stage is also suggested by the measurement by [19] in several reactions of very light residues, as $\mathrm{Be}$ isotopes that are a concern for radioprotection. These isotopes are underpredicted by orders of magnitude by standard codes.

\section{Perspectives}

In order to understand both the mechanism of IMF production and the underprediction of light residues, more constraining experiments are needed. The SPALADIN program [7], in which residues, neutrons and light-charged particles will be measured simultaneously, will help to answer these questions. The excitation energy, $E^{*}$, at the end of the cascade stage will be reconstructed thanks to the multiplicity of neutrons and LCPs. This will allow to determine if the $E^{*}$ is correctly predicted by INCL4. The study of the different decay modes as a function of $E^{*}$ should allow to distinguish between the different possible mechanisms. This is illustrated in Fig. 3 (top) where the excitation energy distribution of events with two fragments with $Z \geqslant 3$ is shown for GEMINI (blue) and SMM (green). In Fig. 3 (bottom), the distribution of the charge difference between the two heaviest fragments
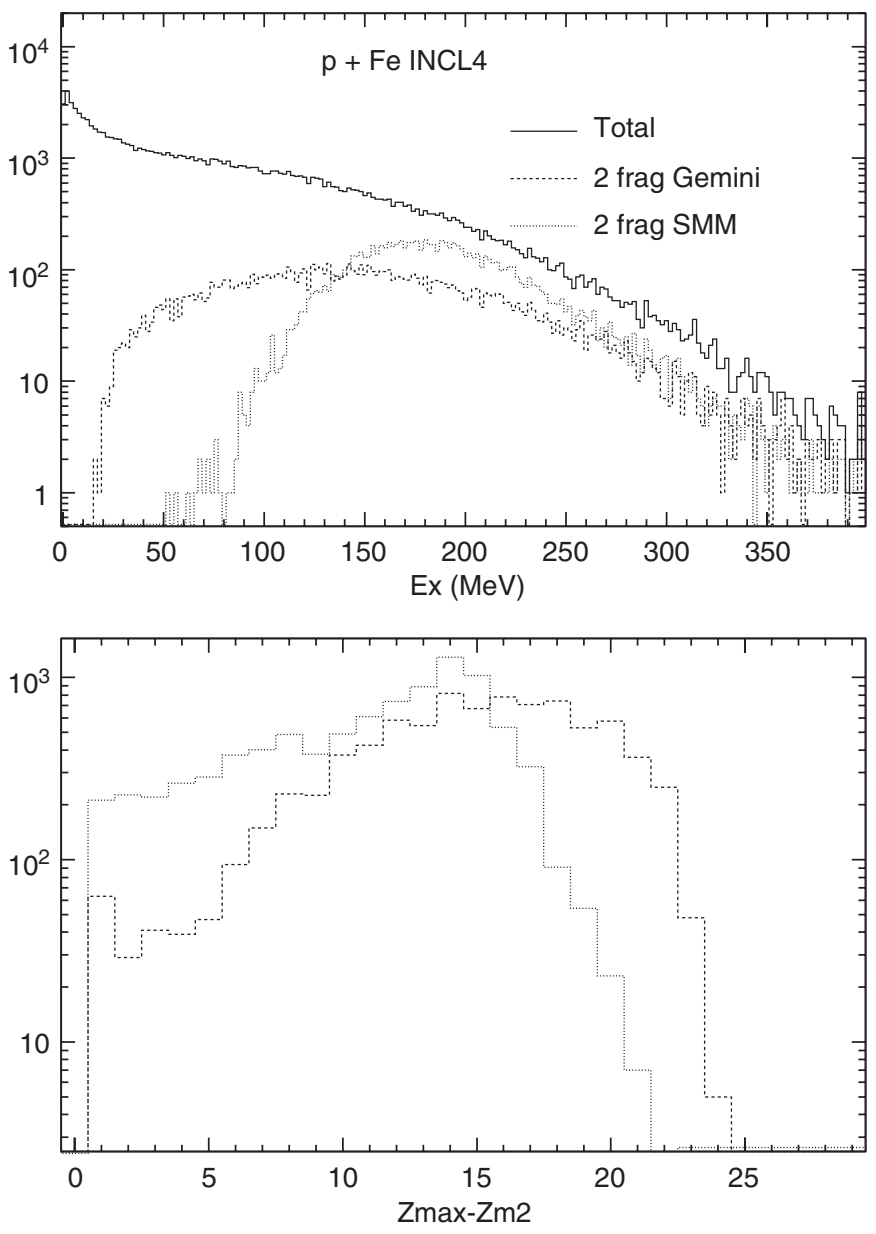

Fig. 3. Top: excitation energy distribution at the end of the cascade calculated with INCL4 for all events (black) and events leading to two fragments with $Z \geqslant 3$ in GEMINI (dashed-dotted) or SMM (dashed line). Bottom: distribution of the charge difference between the two heaviest fragments with the same codes. 
is displayed. In both cases, a clear difference between the predictions of the models is observed that should allow choosing the right mechanism.

As regards the improvement of codes for applications, once the possible missing mechanism will have been clearly identified, it is foreseen to implement it into de-excitation models like ABLA, rather than choosing GEMINI or SMM in order not to loose the agreement on heavy evaporation and fission residues. The cluster formation of LCP in the cascade stage has been found essential to improve helium prediction. This will be included, after some refining, into the INCL4 version in MCNPX. All this work is part of the FP6 EU project EUROTRANS/NUDATRA.

\section{References}

[1] J.P. Meulders, A. Koning, S. Leray (Eds.), HINDAS final report, EU contract FIKW-CT-2000-00031, 2005.

[2] A. Boudard, et al., Phys. Rev. C 66 (2002) 044615.
[3] A.R. Junghans, et al., Nucl. Phys. A 629 (1998) 635.

[4] S.G. Maschnik, et al., AIP Conf. Proc. 768 (2005) 1188.

[5] J.S. Hendricks, et al., MCNPX 2.5.d, Report LA-UR-03-5916.

[6] C.M. Herbach, et al., in: Proceedings of the TRAMU International Workshop, Darmstadt, 1-5 September, 2003, http://www-wnt.gsi.de/ tramu/.

[7] E. Le Gentil, et al., Nucl. Instr. and Meth. A, this conference, doi:10.1016/j.nima.2006.02.037.

[8] H.W. Bertini, Phys. Rev. 131 (1963) 1801.

[9] L. Dresner, ORNL Report ORNL-TM-196, 1962.

[10] T. Aoust, et al., AIP Conf. Proc. 768 (2005) 1572.

[11] D. Hilscher, et al., J. Nucl. Mater. 296 (2001) 83.

[12] R. Michel, et al., Nucl. Instr. and Meth. B 103 (1995) 183.

[13] R.J. Charity, et al., Nucl. Phys. A 483 (1988) 371.

[14] A. Boudard, et al., Nucl. Phys. A 740 (2004) 195; T. Aoust, et al., Nucl. Instr. and Meth. A, this conference.

[15] L. Zanini, et al., AIP Conf. Proc. 768 (2005) 1525.

[16] L. Donadille, et al., J. Nucl. Sci. Technol. (Suppl. 2) (2002) 1194.

[17] C. Villagrasa, et al., AIP Conf. Proc. 768 (2005) 842

[18] J.P. Bondorf, et al., Phys. Rep. 257 (1995) 133.

[19] M. Gloris, et al., Nucl. Instr. and Meth. A 463 (2001) 593. 